\title{
Ultrafast slaving dynamics at the protein-water interface studied with 2D-IR spectroscopy
}

\author{
J. T. King and K. J. Kubarych \\ Department of Chemistry, University of Michigan, Ann Arbor, MI 48109, USA
}

\begin{abstract}
The dynamics of hen egg white lysozyme in D2O/glycerol mixtures is studied using two-dimensional infrared spectroscopy. The hydration dynamics and the protein dynamics are studied simultaneously through vibrational probes attached to the protein surface.
\end{abstract}

\section{Introduction}

The dynamic character of proteins is emerging as a crucial aspect of protein function.[1] The dynamics of proteins are often modeled as a hierarchy of energy and corresponding timescales. Local configurations separated by thermally activated barriers near kBT exchange on a timescale of tens of picoseconds, ultimately facilitating global conformational changes that exchange over significantly larger barriers of several $\mathrm{kBT}$ which exchange on nanosecond to millisecond timescales. Experimental evidence has suggested a strong coupling between the internal dynamics of a protein and the motional fluctuations of the solvent [2], but it has been a challenge to observe directly the coupled protein-water dynamics specifically at the interface. Here, we provide experimental evidence that the hydration layer of water around a protein acts as a coupling agent between the thermal fluctuations of the solvent and the conformational sampling of the protein.

We study hen egg white lysozyme (HEWL), a large globular protein, modified with a metal-carbonyl vibrational probe covalently attached to the His15 residue (Fig. 1).[3] 2D-IR spectroscopy provides the vibrational lifetime (Fig. 2A) and frequency-frequency

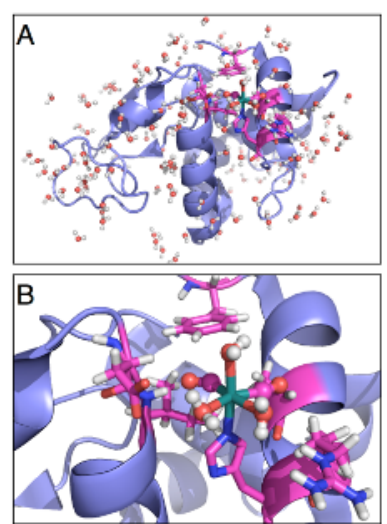

Fig. 1 Structure of HEWL-RC shown with crystallographic waters (A). Local binding environment of the metalcarbonyl vibrational probe at the His15 residue (B). correlation function (FFCF, Fig. 2C) of the $\mathrm{CO}$ vibrational modes of the labeled protein in pure $\mathrm{D} 2 \mathrm{O}$ and $\mathrm{D} 2 \mathrm{O} /$ glycerol mixtures (ranging from $0-80 \%$ glycerol v/v). The vibrational probe is situated at the surface of the protein, and acts as a site-specific sensor of the dynamics at the protein-water interface. The FFCF shows a rapid initial decay on the order of a few picoseconds, which corresponds to the hydration dynamics of the protein, and a significant static offset that is attributed to the slow protein dynamics that are not sufficiently sampled within the experimental window.

This is an Open Access article distributed under the terms of the Creative Commons Attribution License 2.0, which permits unrestricted use, distribution, and reproduction in any medium, provided the original work is properly cited. 


\section{Hydration of proteins in solvent mixtures}

Since lysozyme remains fully hydrated (roughly $5 \AA$ of water around the protein) in glycerol mixtures [4], addition of glycerol offers the possibility of observing both the hydration water and the protein dynamics together as a function of the bulk-viscosity. The strong preferential hydration of proteins in glycerol mixtures allows proteins to function in water/glycerol mixtures containing very small amounts of water (near $1 \%$ water by volume).[5] 2D-IR data supports this picture that lysozyme remains fully hydrated in glycerol mixtures even at low water concentrations. The vibrational lifetime of the vibrational probe is fairly insensitive to the addition of glycerol (Fig. 2A), remaining nearly constant for mixtures ranging from pure D2O to $20 \%$ D2O by volume. Previous work on chaotropic cosolvents has shown that the vibrational lifetime is sensitive to dehydration by a cosolvent.[6] This allows for the bulk-viscosity to be changed by more than two orders of magnitude while leaving the hydration composition unchanged.

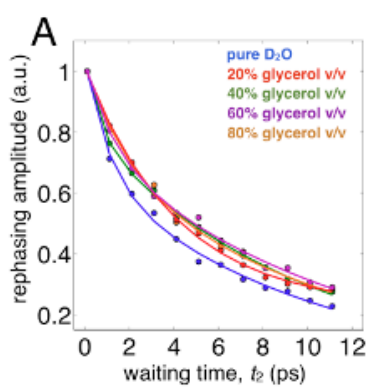

B

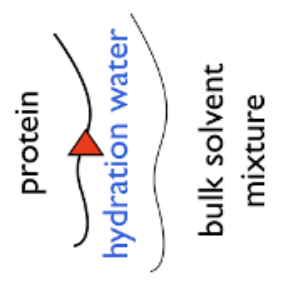

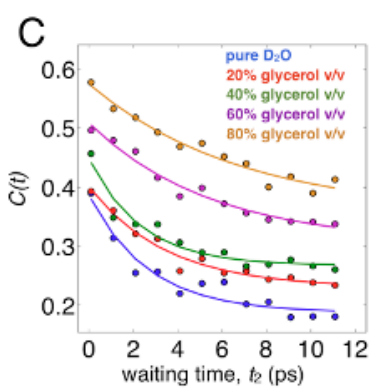

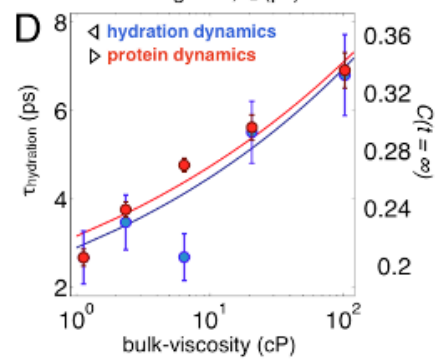

Fig. 2 (A) Vibrational relaxation of HEWL-RC in $\mathrm{D}_{2} \mathrm{O} /$ glycerol mixtures shows no indication of dehydration, consistent with the preservation of a hydrated interface between the protein and the bulk solvent (B); the probe is indicated by a red triangle. (C) The frequency correlation functions extracted from 2D-IR show a fast decay component from the hydration dynamics and a large static offset corresponding to conformational heterogeneity which is not sampled within the experimental window. (D) The hydration dynamics and the protein dynamics are observed to follow a nearly identical slowing trend upon addition of glycerol to the solution, suggesting that the hydration dynamics act to mediate a strong coupling between the protein and the bulk-solvent.

Despite evidence that the protein remains fully hydrated at all glycerol concentrations the FFCFs are found to be extremely sensitive to the addition of glycerol (Fig. 2C). First, the hydration dynamics slow down by nearly a factor of four upon the addition of glycerol (Fig. 2D), decaying with a time constant of 2 ps in pure D2O and nearly 8 ps in $20 \% \mathrm{D} 2 \mathrm{O}(\mathrm{v} / \mathrm{v})$. This slowdown provides evidence that the hydration layer is strongly coupled to the bulk-solvent, despite no evidence for solvent mixing. The effective change in the hydration viscosity is likely due to the confinement imposed both by the protein surface and by the semi-impermeable D2O/gylcerol mixture. With increased glycerol concentration the bulk solution becomes less accessible to the hydration water, which effectively reduces the dimensions available for diffusion.

\section{Coupling of hydration dynamics and protein dynamics to bulk solvent}

The correlation functions show that the hydration water is strongly coupled to the thermal fluctuations of the bulk-solvent. Furthermore the static offset increases upon addition of glycerol, following a nearly identical trend to that found for the hydration dynamics (Fig. 2D). The increase in this static offset corresponds to a slowing in conformational sampling on timescales of hundreds of 
picoseconds or longer. Because there is no direct interaction between glycerol and the protein, this observation suggests that the protein dynamics are modulated by the glycerol through an indirect mechanism where the hydration layer acts to transduce the bulk solution dynamics to the protein surface.

These results provide strong support for slaving models of protein dynamics, which assert that conformational dynamics of proteins are strongly dependent upon the dynamics of the hydration layer and in the bulk solvent.[7] We observe an indirect coupling between the bulk-solvent and the internal dynamics of the protein that is mediated by the hydration layer.

This work highlights the interplay of the protein surface and the hydration environment. Previous work has shown that the protein surface of HEWL is capable of constraining the hydration dynamics through the stifling of hydrogen bond angular jumping events.[6] The dynamics of the hydration water are clearly modulated to some extent by the presence of the protein, and the inhomogenous nature of the protein surface is reflected in the inhomogeneous hydration dynamics surrounding a globular protein.[6] Here, this interplay is further investigated, showing that while the structure of the protein can influence the hydration dynamics, the hydration dynamics can in turn modulate the conformational sampling of the protein. The protein motions that are most sensitive to the solvent viscosity are those that modulate the surface topology, demanding large amplitude hydration water reorganization to accommodate new conformations. More local motions, such as side-chain motions, will likely show a weak solvent dependence, or may even lack a solvent dependence altogether. Hence the ability to sense dynamical coupling at the protein-water interface depends critically on the nature and location of the spectroscopic probe.

[1] K. Henzler-Wildman, D. Kern, Nature 450, 964-972 (2007).

[2] I.J., Finkelstein, A.M. Massari, M.D. Fayer, Biophys. J. 92, 3652-3662 (2007).

[3] T. Santos-Silva, A. Mukhopadhyay, J.D. Seixas, G.J.L. Bernardes, C.C. Romao, M.J. Romao, J. Am. Chem. Soc. 133, 1192-1195 (2010).

[4] K. Gekko, S.N. Timasheff, Biochem. 20, 4667-4676 (1981).

[5] U.R. Desai, A.M. Klibanov, J. Am. Chem. Soc. 117, 3940-3945 (1995).

[6] J.T. King, E.J. Arthur, C.L. Brooks III, K.J. Kubarych, J. Phys. Chem. B 116, 5604-5611 (2012)

[7] P.W. Fenimore, H. Frauenfelder, B.H. McMahon, R.D. Young, Proc. Natl. Acad. Sci. U.S.A. 101, 14408-14413 (2004). 